\title{
Height distribution of the power of 3-min oscillations over sunspots
}

\author{
N. I. Kobanov ${ }^{1}$, D. Y. Kolobov ${ }^{1}$, S. A. Chupin ${ }^{1}$, and V. M. Nakariakov ${ }^{2}$ \\ ${ }^{1}$ Institute of Solar-Terrestrial Physics, Lermontov st., 126a, PO Box 291, 664033 Irkutsk, Russia \\ e-mail: kobanov@iszf.irk.ru \\ 2 Physics Department, University of Warwick, Coventry CV4 7AL, UK \\ Received 23 October 2009 / Accepted 28 September 2010
}

ABSTRACT

\begin{abstract}
Context. The height structure of 3-min oscillations over sunspots is studied in the context of the recently discovered effect of height inversion: over the umbra, the spatial location of the maximum of chromospheric 3-min oscillation power corresponds to the relative decrease in the power of photospheric oscillations.

Aims. We investigate whether the height inversion of the power of 3-min oscillations is a common feature of the spatial structure of the oscillations for the majority of sunspots.

Methods. Spectrogram sequences of H $\alpha 6563 \AA$ and Fe I $6569 \AA$ over sunspots, acquired with very high cadency (about 2 s or better) are obtained. The distribution of the oscillation power of the line-of-sight velocity signal is studied by using methods of wavelet frequency filtration and Fourier analysis.

Results. The effect of the height inversion is found in 9 of 11 analyzed active regions. The interpretation of this effect is possibly connected to both the decrease in the level of photosphere in sunspot umbrae and the magnetic field topology.
\end{abstract}

Key words. sunspots - Sun: oscillations - Sun: chromosphere - Sun: photosphere

\section{Introduction}

Powerful 3-min oscillations of the line-of-sight (LOS) velocity, detected in the Doppler shifts of the chromospheric spectral lines above sunspots have been subject to intensive studies for several decades (Beckers \& Tallant 1969; Giovanelli 1972; Zirin \& Stein 1972; Lites 1992). The interest in this phenomenon is justified for several important reasons. First of all, it is connected to the transfer of the energy of photospheric motions by these waves to the corona. There is observational evidence of 3-min field-aligned compressible waves in the corona over sunspots, usually seen in $171 \AA$ and $195 \AA$ passbands of TRACE (see, e.g. De Moortel 2006), which may be associated with the leakage of the 3-min sunspot oscillations. As the waves are interpreted as slow magnetoacoustic waves that propagate almost parallel to the magnetic field, their path highlights the magnetic connectivity of the photospheric and coronal plasmas. In addition, these waves are a useful tool for probing solar coronal plasmas in the quickly developing research field of coronal seismology (King et al. 2003; Wang et al. 2007). Moreover, the relationship between 3-min oscillations in sunspots and the appearance of a similar periodicity in flaring energy releases has been established (Sych et al. 2009). Another useful application of 3-min oscillations is in attempting to reveal the internal, sub-photospheric structuring of sunspots (e.g. Zhugzhda 2008).

3-min sunspot oscillations are presently studied in the optical band by both filtering and spectral methods, as well as in radio and UV bands (Gelfreikh et al. 1999; O'Shea et al. 2002; Rouppe van der Voort et al. 2003; Bloomfield et al. 2007; Nagashima et al. 2007; Jess et al. 2007; Tziotziou et al. 2007; Balthasar \& Schleicher 2008). Kobanov \& Makarchik (2004); Kobanov et al. (2006); Bloomfield et al. (2007) demonstrated that 3-min oscillations in the umbral chromosphere are not the source of the running penumbral waves and concluded that the apparently horizontally propagating waves correspond to the "visual pattern" scenario.

Another identified peculiarity in the behaviour of 3-min oscillations has been the effect of height inversion (Kobanov et al. 2008): at the chromospheric level, the spatial localisation of the maximum of 3-min oscillation power, usually over the umbra, often corresponds to the minimum of these oscillations at the photospheric level. One would expect that the spatial distribution of 3-min oscillations at both heights is similar, although at the photospheric level the oscillation power in the umbra is lower than in the penumbra and adjacent regions. This finding is consistent with the results of previous studies. In particular, it was pointed out that in the umbra, at the photospheric height, 3-min oscillations are either not detected at all (Balthasar et al. 1987) or of very low amplitude relative to the level of noise (Lites \& Thomas 1985). We note that the suppression of the photospheric oscillation power in active regions has also been detected by the helioseismological methods (Braun \& Duvall 1990; Braun 1995; Nicholas et al. 2004). The analysis of the brightness fluctuations observed in the G-band with Hinode/SOT detected the decrease in the broadband oscillation power in central parts of sunspots (Nagashima et al. 2007).

The aim of this paper is to perform a comparative analysis of 3-min oscillations for a number of sunspots, demonstrate that the height inversion is a statistically significant and reproducible effect, and contribute to its interpretation. Our approach is based on the use of high cadence optical data, with the time resolution of about $2 \mathrm{~s}$, and upon the consideration of 3-min oscillations in a narrow band, $4.7-6.7 \mathrm{mHz}$. The paper is organised as follows. In the next section, we describe the instrument used in the observations and data reduction. In Sect. 3, we present the results obtained. The results obtained are summarised in the conclusions. 


\section{The instrument and data reduction}

We use data obtained with the Horizontal Solar Telescope at the Sayan Solar Observatory, Russia (Kobanov et al. 2009). The diameter of the mirror of the instrument is $80 \mathrm{~cm}$, hence the theoretically possible spatial resolution is $0.2^{\prime \prime}$. However, because of the Earth's atmospheric conditions the resolution is usually about 1 ". The guiding system carries out targeting and object capturing with a precision no worse than $1^{\prime \prime}$. In observations, an astronomical CCD of the resolution $256 \times 1024$ pixels was used. The spectral snapshot contains information about a spatial region of the size $63 \times 0.5^{\prime \prime}$, defined by the slit of the spectrograph. For each spatial element, we obtain the spectrum of the width of $8.5 \AA$ (for the spectrograph dispersion of the fifth order in the vicinity of the $\mathrm{H} \alpha 6563 \AA$ line). The image was rotated in a way to position the spectrograph slit at the sunspot centre in either the north-south or east-west directions (see Fig. 1), by using a Dove prism in front of the spectrograph. The duration of observational sequences was about one hour with a cadence time of about two seconds. The majority of the observational sequences were recorded with the use of a deflector (Kobanov 2001), which provided us with information about the LOS velocity, spectral line intensity, and the LOS component of the photospheric magnetic field (by the Fe I $6569 \AA$ line) simultaneously.

The information was recorded in a digital data cube of the following structure. Two dimensions corresponded to the pixel number along the spectrograph slit and dispersion (hence the spatial and spectral information), while the third dimension contained the intensity of the emission at the certain instant of time. A dedicated software package was then applied to reduce the data cube to a two-dimensional array. The array contained the distribution of the intensity and the LOS components of the velocity and magnetic field along the slit at each instant of time. In the analysis of oscillatory processes, the time sequences of each spatial pixel were studied by the Fourier and wavelet techniques. The wavelet analysis was performed according to the methodology described in Torrence \& Compo (1998) with the 6th-order Morlet basis function.

The LOS component of the velocity was obtained by the lambdameter technique (Rayrole 1967). In this method, the spectral position of a chosen line is determined by two virtual slits. The distance between the slits is known and remains constant during the measurements. Initially, the slits are situated at equal distances from the line centre, hence have equal intensities. If in the next spectrogram the line is displaced, the intensities measured by the slits differ. Displacing the slits to make their intensities equal allows one to determine the new location of the line. Since this method is based upon the determination of the relative position of the line, it is not affected by the variation in the intensity in time, from one spectrogram to another.

In the processing of the observational sequences obtained with the deflector, the LOS velocity is determined by the Doppler formula directly from the displacement of $\mathrm{H} \alpha$ line, as the splitting of the line by the deflector is insignificant. In the case of another line used in this study, Fe I, it is completely split by the deflector into the $\sigma_{-}$and $\sigma_{+}$components. The LOS velocity is proportional to the sum of the displacements of these components, while the magnetic field is proportional to the difference in the displacements. The magnetic field is then calculated according to the Zeeman effect for a simple triplet. Telluric line near $\mathrm{H} \alpha$ was used to eliminate the spectrograph noise.

A possible error in the determination of the line position may be related to the asymmetry of the line shape. The asymmetry can be caused by blending of the $\mathrm{H} \alpha$ line, or by the variation

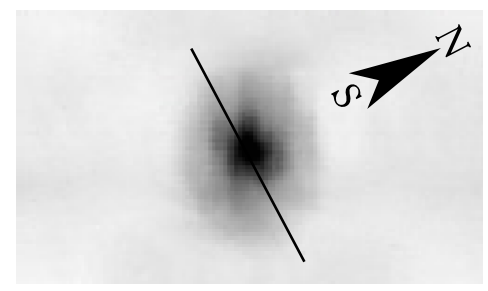

Fig. 1. An example of the spectrograph slit orientation in the observations of the sunspot NOAA 791 (the scale of the slit is not kept).

Table 1. Observational sequences studied in this paper.

\begin{tabular}{ccccc}
\hline \hline NOAA & Solar coordinates & Date & Start time, UT & $P_{\mathrm{p}} / P_{\mathrm{c}}$ \\
\hline 051 & S17E01 & 02.08 .2002 & $00: 45$ & $0.5 / 12$ \\
051 & S16E10 & 01.08 .2002 & $06: 52$ & $0.8 / 12.9$ \\
$105 \mathrm{~L}$ & S08E39 & 11.09 .2002 & $01: 21$ & $0.6 / 9.2$ \\
613 & S09W03 & 20.05 .2004 & $03: 25$ & $0.6 / 12.2$ \\
621 & S14W06 & 04.06 .2004 & $05: 43$ & - \\
657 & N10W03 & 13.08 .2004 & $01: 44$ & $0.6 / 7.3$ \\
$661 \mathrm{~L}$ & N06E42 & 15.08 .2004 & $23: 57$ & $0.7 / 3.7$ \\
$661 \mathrm{~L}$ & N07E20 & 18.08 .2004 & $00: 16$ & $0.7 / 11.4$ \\
$661 \mathrm{~F}$ & N07E19 & 18.08 .2004 & $01: 01$ & $1.2 / 38.2$ \\
$791 \mathrm{~L}$ & N13E21 & 26.07 .2005 & $01: 37$ & $0.5 / 5.1$ \\
$791 \mathrm{~L}$ & N13E20 & 26.07 .2005 & $03: 27$ & $2.1 / 15.6$ \\
$791 \mathrm{~L}$ & N13E08 & 27.07 .2005 & $00: 53$ & $0.3 / 26.5$ \\
$791 \mathrm{~L}$ & N13E06 & 27.07 .2005 & $01: 26$ & $0.5 / 16.3$ \\
$791 \mathrm{~L}$ & N13E06 & 27.07 .2005 & $03: 06$ & $0.6 / 25.3$ \\
$791 \mathrm{~L}$ & N13E05 & 27.07 .2005 & $04: 46$ & $0.4 / 33.3$ \\
794 & S11E32 & 04.08 .2005 & $02: 47$ & $0.7 / 11$ \\
794 & S11E32 & 04.08 .2005 & $03: 38$ & $1.1 / 18.6$ \\
794 & S11E05 & 05.08 .2005 & $01: 22$ & $0.8 / 93$ \\
810 & N10E23 & 21.09 .2005 & $02: 45$ & $0.9 / 9.2$ \\
810 & N10E23 & 21.09 .2005 & $06: 48$ & $0.8 / 6.3$ \\
810 & N09W07 & 23.09 .2005 & $07: 15$ & $1.5 / 9.2$ \\
$886 \mathrm{~L}$ & N07E24 & 25.05 .2006 & $00: 47$ & $0.8 / 3.5$ \\
$886 \mathrm{~L}$ & N08E02 & 26.05 .2006 & $04: 25$ & $0.7 / 4.7$ \\
$963 \mathrm{~L}$ & S07E62 & 08.07 .2007 & $23: 37$ & $0.5 / 4$ \\
\hline
\end{tabular}

in the LOS speeds between the heights covered by the optically thick $\mathrm{H} \alpha$ line. However, as in this study we consider not the absolute values of the LOS speeds, but the frequencies of their time variations, the latter effect not being likely to cause significant error. In this paper, we did not discuss the results of the magnetic field measurements.

\section{Results}

In our study, we analysed 11 active regions (AR) observed during 2002-2007. A list of the AR with their locations on the solar disk, the time of the observation, and results is presented in Table 1. The duration of each sequence is about one hour, where $P_{\mathrm{p}}$ and $P_{\mathrm{c}}$ are the ratios of the mean power of the 3 min oscillations in the spot umbra to the mean power in the penumbra for the photospheric $\left(P_{\mathrm{p}}\right)$ and chromospheric $\left(P_{\mathrm{c}}\right)$ level correspondingly.

The spatial localisation of the 3-min oscillations of the LOS velocity has been studied using both Fourier and wavelet analyses. In both cases, the analysed spectral interval was taken to be from 4.7 to $6.7 \mathrm{mHz}$. Figure 2 shows a typical wavelet spectrum that gives the time-spatial dynamics of the 3-min oscillation, as well as the time-averaged spatial distribution of the oscillation power.

Our analysis demonstrated that at the chromospheric level in all considered sunspots except the small sunspot NOAA 621, the maxima of 3-min oscillation power are situated near the centres 

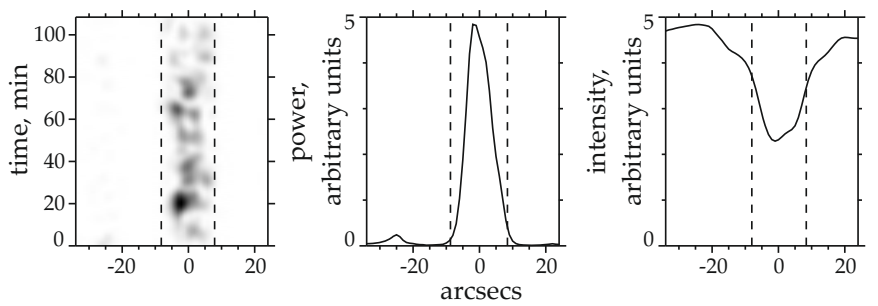

Fig. 2. The distribution of the wavelet power of the LOS velocity in the range 4.7-6.7 $\mathrm{mHz}$ in the chromosphere of NOAA 810 observed in $\mathrm{H} \alpha$. Left panel: time-spatial dynamics of the 3-min oscillations. Central panel: profile of the time-averaged spatial distribution of the wavelet power. Right panel: the profile of the intensity observed in the continuum. The vertical dashed lines indicate the umbra-penumbra boundary.
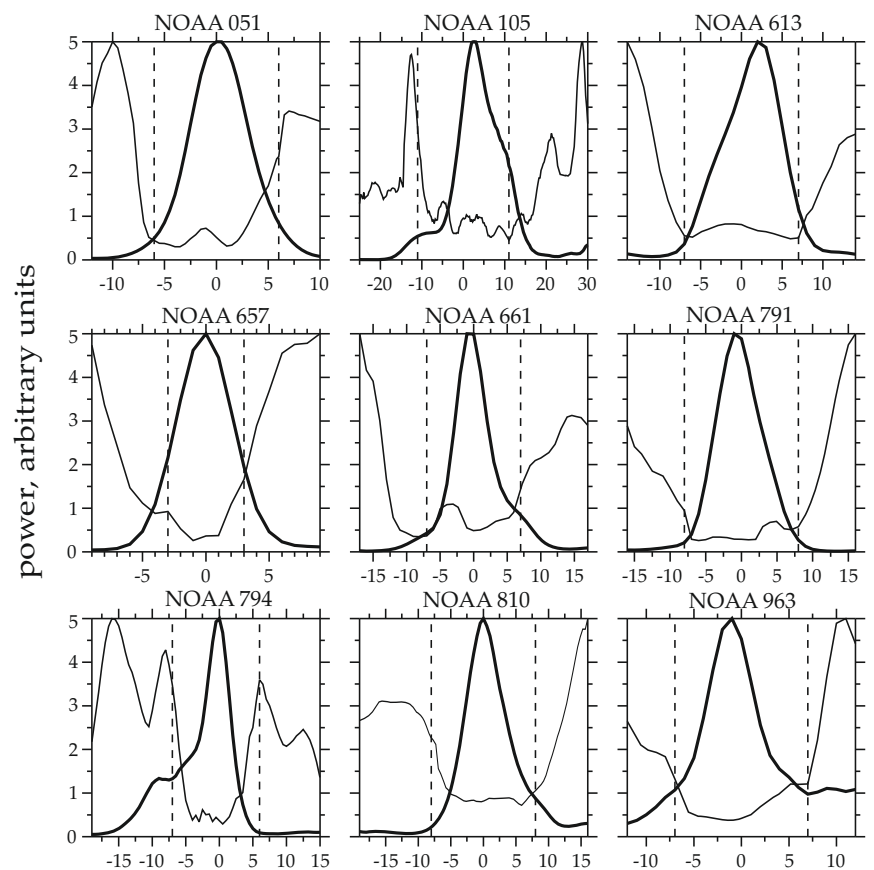

NOAA 791
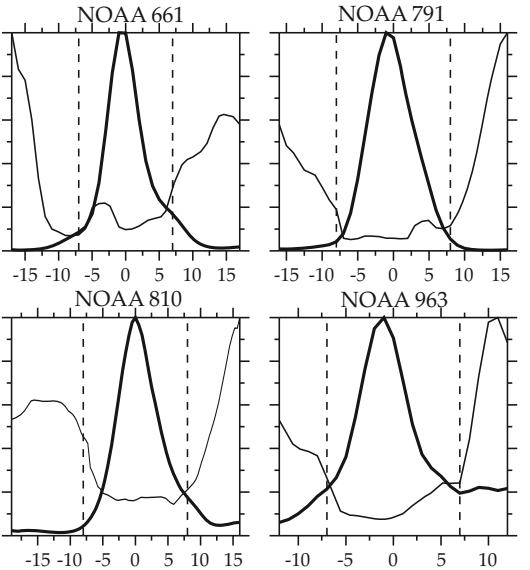

arcsecs

Fig. 3. The effect of the height inversion for the power of 3-min oscillations in sunspot umbrae. The panels show time-averaged spatial profiles of 3-min oscillations of the LOS velocity measured in different active regions. Thin lines correspond to the photospheric signal ( $\mathrm{Fe} \mathrm{I})$, and the thick curves to the chromospheric signal $(\mathrm{H} \alpha)$. The vertical dashed lines indicate the umbra-penumbra boundary.

of the umbrae. At the photospheric level, in the most considered time series, these regions were found to correspond to the suppression of the 3-min oscillation power (see Fig. 3). The criterion used in the detection of the effect of the height inversion is the occurrence of a local minimum in the spatial distribution of the photospheric narrowband power and its absence at the chromospheric level. The spatial coordinates in all plots of Fig. 3 were restricted by the external boundaries of the penumbra. The effect can be quantified by the ratio of the mean power in the umbra (averaged over the whole umbra) to the mean power in the penumbra (averaged over the whole penumbra). In the chromosphere, this ratio was found to be greater than one, while in the photosphere it is smaller than one (see $P_{\mathrm{p}} / P_{\mathrm{c}}$, Table 1 ). We analysed 24 time series from 11 active regions. In 18 series, we found the effect of height inversion, in 3 series the situation was the opposite, and for 2 series we derived uncertain results (the photospheric mean power ratio was 0.9-1.1). One time series of NOAA 621 shows the absence of the 3-min LOS velocity oscillations in the chromosphere. We should also point out that in
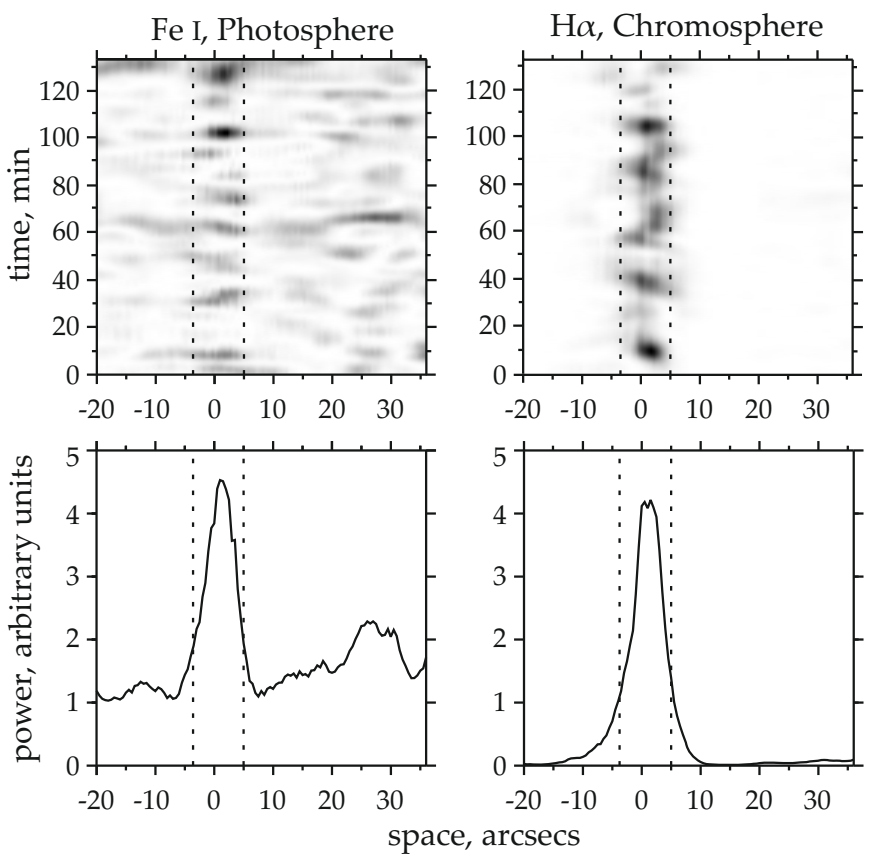

Fig. 4. The absence of the height inversion effect for the leading sunspot of NOAA 791 in the observational sequences on 2005 July 26, 03:275:40 UT. In the same sunspot, the effect of the height inversion is presented in other five time series (one of them is shown in Fig. 3). The figure shows the spatial distribution of the wavelet power of the LOS oscillations in the band 4.7-6.7 mHz. Top panels: time-spatial dynamics of the wavelet spectra. Bottom panels: time-averaged profiles of the wavelet spectra. The vertical dashed lines indicate the umbra-penumbra boundary.

certain time intervals in some sunspots the maximum of 3-min oscillations at the photospheric level can be found in the umbra. For example, for NOAA 791, in six analysed time sequences, the effect of the height inversion was found in five cases, while in one case the photospheric maximum of the 3-min oscillations was localised in the umbra, hence exactly coincident with the chromospheric maximum (see Fig. 4). Wavelet spectra of the photospheric and chromospheric signals are shown in the upper panels. At first glance, the increases and decreases in the oscillation power at those heights seem to occur simultaneously. However, the detailed phase analysis discussed below does not support this impression.

We are thus inclined to conclude that in the majority of the observed cases the spatial location of the maxima of 3-min chromospheric oscillations correspond to the suppression of these oscillations at the photospheric level. One possible interpretation of this effect may be the decrease in the photospheric level observed in Fe I $6569 \AA$ (the Wilson depression), as it is known that the amplitude of acoustic oscillations decreases in deeper regions (Lites 1992; Sigwarth \& Mattig 1997). The question is whether the difference in heights, associated with the Wilson depression, is responsible for the observed decrease in the oscillation amplitude by a factor of two.

According to Balthasar \& Woehl (1983) and Watson et al. (2009), the depression $(\tau=1)$ of the umbral photosphere with respect to the penumbra (the Wilson effect) is about $700 \mathrm{~km}$. This difference in height can lead to the decrease in the 3-min oscillation amplitude by a factor of 2 (Lites et al. 1998). This decrease is consistent with the 3-min LOS velocity power distribution at the photospheric level (Fig. 3), at least within the order of the magnitude. 
Parnell \& Beckers (1969) used the formation depth of the line Fe I 6569 to study the granulation. They determined the upper limit to the line formation height to be $250 \mathrm{~km}$. According to the broadly used atlas of the solar spectrum (Moore et al. 1966), this line is suppressed in sunspots, hence one can assume that its optical thickness in the umbra is not higher. In the Fe I 6569 line, the Wilson effect is as pronounced as in the continuum.

The observed effect may also be caused by the topology of the sunspot magnetic field. If we assume that a horizontally extended source of 3-min oscillations is situated below the photosphere, in the umbra, the oscillations in the form of slow magnetoacoustic waves would be guided by the vertical magnetic field lines upwards and reach the chromosphere. At the edge of the umbra and in the penumbra, the magnetic field lines are inclined from the vertical direction, preventing the free propagation of the short period waves (Bogdan \& Judge 2006). Thus, under the canopy 3-min waves are able to propagate in the vertical direction up to the magnetic dome only. Finsterle et al. (2004) pointed out that the $7-\mathrm{mHz}$ oscillations are reflected by the active region canopy. The partial reflection of the waves from the magnetic dome can lead to the observed accumulation of the wave energy at the photospheric level under the regions with a horizontal magnetic field. This may explain the relative increase in the 3 -min photospheric oscillation power in the regions outside the umbra, and the corresponding decrease in the chromospheric oscillation power in these regions.

Moreover, it is necessary to consider a possible contribution of the "power halo" (Lindsey \& Braun 1999; Braun \& Lindsey 1999). Our observations were focused on the sunspots, and, unfortunately, studying the sunspots' vicinities is beyond the scope of the data for most time series.

In the majority of the observational sequences, the power of photospheric 3-min oscillations in the umbra was low, thus it was impossible to estimate the phase delay between the photospheric and chromospheric oscillatory motions in the umbra. However, the above-mentioned unusual observational sequences of NOAA 791, containing the umbral maximum of the photospheric 3-min oscillations, allows us to carry out the analysis of the phase shifts between the signals at different heights. Unfortunately, the direct comparison of the photospheric and chromospheric signals does not provide us with any conclusive results. Figure 5 shows that there is no stable phase difference between narrowband photospheric and chromospheric signals. In some time intervals (e.g. 45-50 min), the chromospheric signal even precedes the photospheric signal. There are also time intervals (e.g. 112-130 min) when the time lag gradually increases from $20 \mathrm{~s}$ to $140 \mathrm{~s}$. The correlation between the signals for the whole time series is presented in the right panel of Fig. 5.

A similar negative result comes from the comparison of the power maxima of the photospheric and chromospheric signals (Fig. 6). The detuning of the signals is so pronounced that one has the impression that the photospheric and chromospheric signals are disconnected. Thus, the data obtained does not allow us to measure the phase lag between the photospheric and chromospheric 3-min oscillations directly, to derive the phase speed. However, the average time lag between the appearance of the maxima of the oscillation power at those two heights could be measured and is about $150 \mathrm{~s}$. Taking the distance between the heights of observations to be $2000 \mathrm{~km}$ (Vernazza et al. 1981; White \& Wilson 1966), we obtain the vertical group speed of about $13 \mathrm{~km} \mathrm{~s}^{-1}$.

On the other hand, taking into account the correlogram shown in the right panel of Fig. 6, the group speed can be estimated as $6 \mathrm{~km} \mathrm{~s}^{-1}$. This discrepancy is due to the ambiguity

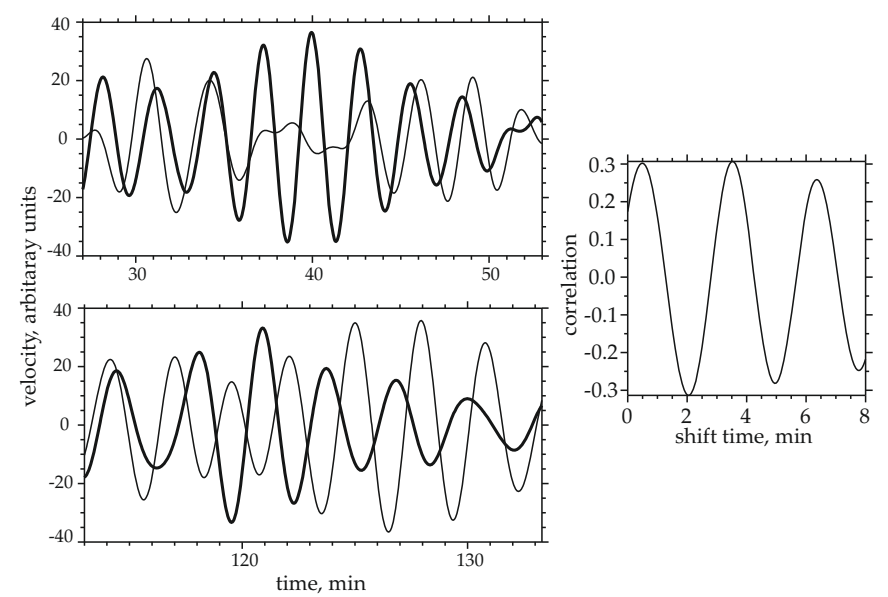

Fig. 5. Left panel: comparison of phases of chromospheric (thick line) and photospheric (thin line) narrowband $(4.7-6.7 \mathrm{mHz})$ LOS velocity signals in the centre of the umbra of NOAA 791 (the leading spot) in different time intervals. Right panel: correlation between the chromospheric and photospheric signals of the LOS velocity for the whole time series.

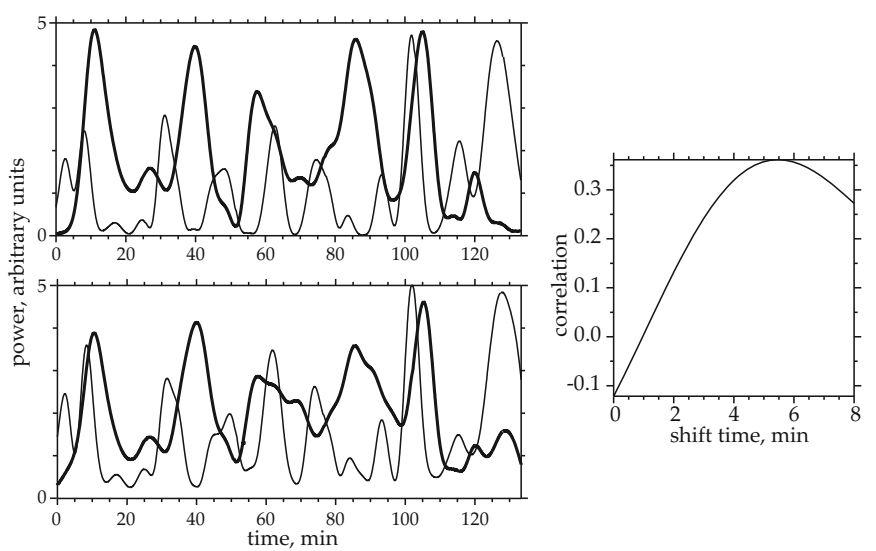

Fig. 6. The time variation in the power of the chromospheric (thick line) and photospheric (thin line) narrowband $(4.7-6.7 \mathrm{mHz}$ ) LOS velocity signals in the umbra of the leading spot of NOAA 791. The upper panel shows the signals of the spatial element of $1^{\prime \prime}$ at the centre of the umbra. The bottom panel shows the signals averaged over the $10^{\prime \prime}$ region in the umbra. Right panel shows the correlation between the signals presented in the upper panel.

of the phase connection between the photospheric and chromospheric signals. Moreover, the observed correlation coefficient is rather low, about 0.35 , and is observed only in one case. Thus, the present study does not allow us to make confident and statistically-significant conclusions about the wave connectivity at the photospheric and chromospheric levels, based upon the correlation analysis. One possible reason for the apparent out-ofphase behaviour of the photospheric and chromospheric oscillations may be the partial reflection of the slow magnetoacoustic waves from the density gradient over the umbra, which causes a complicated time-dependent superposition of the propagating and standing waves in the photosphere.

\section{Conclusions}

We have analysed the spatial distribution of narrowband 3-min oscillations of the LOS velocity at the photospheric and chromospheric levels in eleven sunspots. The observations dedicated to this study were carried out with the $80 \mathrm{~cm}$ Horizontal Solar Telescope at the Sayan Solar Observatory in the Fe I $6569 \AA$ and 
H $\alpha 6563 \AA$ lines, respectively. The spectral resolution of the instrument is $8.5 \mathrm{~m} \AA$. The cadence time of the measurements was about $2 \mathrm{~s}$. At the chromospheric level in ten of the eleven considered sunspots, the maxima of 3-min oscillation power were found to be situated near the centres of the sunspot umbrae. In nine of the eleven sunspots at the photospheric level, the 3-min oscillation power was found to be suppressed in the umbra relative to the peripheral regions.

Thus, in the umbra, the chromospheric oscillations have higher power than outside, while, in contrast, the photospheric oscillations in the umbra have systematically lower amplitude than in the surrounding regions. This effect may be termed a height inversion of 3-min oscillations in sunspots. Phase shifts between narrowband 3-min amplitude photospheric and chromospheric signals were found to vary in time, as well as between the maxima of their power. The effect of height inversion may be attributed to the wave reflection caused by the inclined magnetic fields in the peripheral regions of sunspots or to the Wilson depression of the photospheric level in the umbra. However, detailed theoretical study of these phenomena is needed.

Acknowledgements. The work is supported in part by the grant RFBR 0802-91860-KO_a, the Royal Society British-Russian International Joint Project, grant 10-02-00153-a and grant of Federal Agency for Science and Innovation (State Contract 02.740.11.0576). Wavelet software was provided by C. Torrence and G. Compo, and is available at http://paos.colorado.edu/research/ wavelets. We would like to thank anonymous referee for the detailed consideration of our paper and for the constructive comments.

\section{References}

Balthasar, H., \& Schleicher, H. 2008, A\&A, 481, 811

Balthasar, H., \& Woehl, H. 1983, Sol. Phys., 88, 71

Balthasar, H., Kueveler, G., \& Wiehr, E. 1987, Sol. Phys., 112, 37

Beckers, J. M., \& Tallant, P. E. 1969, Sol. Phys., 7, 351

Bloomfield, D. S., Lagg, A., \& Solanki, S. K. 2007, ApJ, 671, 1005

Bogdan, T. J., \& Judge, P. G. 2006, Roy. Soc. London Philos. Trans. Ser. A, 364, 313
Braun, D. C. 1995 , ApJ, 451, 859

Braun, D. C., \& Duvall, Jr., T. L. 1990, Sol. Phys., 129, 83

Braun, D. C., \& Lindsey, C. 1999, ApJ, 513, L79

De Moortel, I. 2006, Roy. Soc. London Philos. Trans. Ser. A, 364, 461

Finsterle, W., Jefferies, S. M., Cacciani, A., Rapex, P., \& McIntosh, S. W. 2004, ApJ, 613, L185

Gelfreikh, G. B., Grechnev, V., Kosugi, T., \& Shibasaki, K. 1999, Sol. Phys., 185,177

Giovanelli, R. G. 1972, Sol. Phys., 27, 71

Jess, D. B., Andić, A., Mathioudakis, M., Bloomfield, D. S., \& Keenan, F. P. 2007, A\&A, 473, 943

King, D. B., Nakariakov, V. M., Deluca, E. E., Golub, L., \& McClements, K. G. 2003, A\&A, 404, L1

Kobanov, N. I. 2001, Instr. Exp. Tech., 4, 110

Kobanov, N. I., \& Makarchik, D. V. 2004, A\&A, 424, 671

Kobanov, N. I., Kolobov, D. Y., \& Makarchik, D. V. 2006, Sol. Phys., 238, 231

Kobanov, N. I., Kolobov, D. Y., \& Chupin, S. A. 2008, Astron. Lett., 34, 133

Kobanov, N. I., Kolobov, D. Y., Sklyar, A. A., Chupin, S. A., \& Pulyaev, V. A. 2009, Astron. Rep., 53, 957

Lindsey, C., \& Braun, D. C. 1999, ApJ, 510, 494

Lites, B. W. 1992, in NATO ASIC Proc. 375, Sunspots. Theory and Observations, ed. J. H. Thomas, \& N. O. Weiss, 261

Lites, B. W., \& Thomas, J. H. 1985, ApJ, 294, 682

Lites, B. W., Thomas, J. H., Bogdan, T. J., \& Cally, P. S. 1998, ApJ, 497, 464

Moore, C. E., Minnaert, M. G. J., \& Houtgast, J. 1966, The solar spectrum 2935 A to 8770 A, ed. C. E. Moore, M. G. J. Minnaert, \& J. Houtgast

Nagashima, K., Sekii, T., Kosovichev, A. G., et al. 2007, PASJ, 59, 631

Nicholas, C. J., Thompson, M. J., \& Rajaguru, S. P. 2004, Sol. Phys., 225, 213

O'Shea, E., Muglach, K., \& Fleck, B. 2002, A\&A, 387, 642

Parnell, R. L., \& Beckers, J. M. 1969, Sol. Phys., 9, 35

Rayrole, J. 1967, Annales d'Astrophysique, 30, 257

Rouppe van der Voort, L. H. M., Rutten, R. J., Sütterlin, P., Sloover, P. J., \& Krijger, J. M. 2003, A\&A, 403, 277

Sigwarth, M., \& Mattig, W. 1997, A\&A, 324, 743

Sych, R., Nakariakov, V. M., Karlicky, M., \& Anfinogentov, S. 2009, A\&A, 505,791

Torrence, C., \& Compo, G. P. 1998, Bull. Amer. Meteor. Soc., 79, 1, 61, 79

Tziotziou, K., Tsiropoula, G., Mein, N., \& Mein, P. 2007, A\&A, 463, 1153

Vernazza, J. E., Avrett, E. H., \& Loeser, R. 1981, ApJS, 45, 635

Wang, T., Innes, D. E., \& Qiu, J. 2007, ApJ, 656, 598

Watson, F., Fletcher, L., Dalla, S., \& Marshall, S. 2009, Sol. Phys., 260, 5

White, O. R., \& Wilson, P. R. 1966, ApJ, 146, 250

Zhugzhda, Y. D. 2008, Sol. Phys., 251, 501

Zirin, H., \& Stein, A. 1972, ApJ, 178, L85 